\title{
Uso do fator de condição alométrico e de fulton na comparação de carpa (Cyprinus carpio), considerando os sexos e idade
}

\section{Use of the alometric and the fulton condition factors to compare the carp (Cyprinus carpio) considering sexes and ages}

\author{
Marco Antonio da Rocha ${ }^{1 *}$; Edson Luis de Azambuja Ribeiro ${ }^{1}$; \\ Ivone Yurika Mizubuti ${ }^{1}$; Leandro das Dores Ferreira da Silva ${ }^{1}$; \\ Julian Cristina Borosky²; Karina Cristina Puggesi Rubin ${ }^{2}$
}

\section{Resumo}

\begin{abstract}
Em peixes, a relação entre o peso corporal e o comprimento corporal total permite calcular o fator de condição, o qual determina o seu grau de desenvolvimento. O experimento foi realizado na Estação de Piscicultura da Universidade Estadual de Londrina, a amostra era constituída de 65 peixes, sendo 31 machos e 34 fêmeas, oriundos da mesma desova e criados nas mesmas condições ambientais. Com a avaliação do peso e do comprimento corporal total em idades de 60;120;180 e 240 dias determinou-se a taxa de crescimento médio e os fatores de condição de Fulton e alométrico. Os resultados mostraram diferença no grau de desenvolvimento entre sexos. $\mathrm{O}$ grau de desenvolvimento nas fêmeas foi maior que nos machos, a exceção na idade 240 dias. De acordo com os resultados obtidos, o escore condição alométrico é o mais indicado.
\end{abstract}

Palavras-chave: Peixes, carpa, fator de condição

\begin{abstract}
In fish, the relation beteween body weight and total length allows us to calculate the condition factor, which determines its grade of development. The experiment was conducted at the Fishery Station of the State University of Londrina. The sample was constituted of 65 fishes, 31 males and 34 females. Fishes came from the same hatch and were raised at the same enviromental condition. Using the weights and the total body lengths at ages 60,120,180 and $240 \mathrm{~d}$, the alometric and the Fulton condition factors, were determined. The results indicate that the two condition factors presented differences in the grade of development between sexes and within sex. The grade of development in females was greater than males, with the exception of the age $240 \mathrm{~d}$. As the relation between body weight and body length in carp is not isometric, the alometric condition score is the most indicated.
\end{abstract}

Key words: Condition factor, fish, carp

\footnotetext{
1 Professores associados do departamento de Zootecnia da Universidade Estadual de Londrina, Londrina, Paraná, Brasil. marco@uel.br

2 Acadêmicas do Curso de Medicina Veterinária da Universidade Estadual de Londrina, Londrina, Paraná, Brasil.

* Autor para correspondência
} 


\section{Introdução}

A piscicultura como atividade econômica tem crescido muito nos últimos anos no Brasil. O incremento maior desta atividade deve ser feito através dos conhecimentos a respeito das espécies a serem exploradas. Desta forma, as pesquisas básicas e aplicadas devem ser incentivadas para que a atividade tenha uma rentabilidade maior.

O crescimento é um fator importante na criação dos peixes e que pode ser medido através do peso, do comprimento corporal em função da idade. De acordo com Royce (1972), o crescimento pode ter uma influência endógena representado pela herança e ação de hormônios e exógena que é representados por um complexo de fatores ambientais, sendo provavelmente a quantidade e qualidade dos alimentos o mais importante.

A relação entre o peso e o comprimento corporal pode apresentar condições de crescimento isométrico quando $b=3,0$, isto é, o peso aumenta com o comprimento elevado ao cubo. Por outro lado, quando $\mathrm{b}<3,0$ o incremento é devido ao peso, e quando $\mathrm{b}>$ 3,0 o incremento é devido ao comprimento.

Os valores do coeficiente de regressão (b) para peixes podem assumir valores entre 2,5 e 4,0 (LE CREN, 1951) ou 2,0 e 3,5 (ROYCE, 1972). Geralmente os valores de b giram em torno de 3,0 (crescimento isométrico).

A relação entre o peso e o comprimento corporal permite calcular um parâmetro que determina o grau de bem estar do peixe, e é o que se chama de fator de condição. Desta forma, a nutrição, doenças e contaminantes são altamente interrelacionados com a criação do peixe. $\mathrm{O}$ aporte nutricional insuficiente pode alterar o fator de condição, conforme Ratz e Lloret (2003). O fator de condição permite comparações entre populações de peixes, que estão submetidas a diferentes condições de clima, temperatura, alimentação, densidade, etc.

A estimativa do fator de condição pode ser feita através do fator de condição de Fulton e do fator de condição alométrico. O fator de condição de Fulton assume que a relação peso/comprimento é isométrico. Por outro lado, o fator de condição alométrico considera que as várias espécies de peixes podem ter diferentes relações de peso/comprimento.

O objetivo deste trabalho foi o de estudar o crescimento da carpa (Cyprinus carpio) em condições normais de manejo, considerando os sexos e idades.

\section{Material e Métodos}

O experimento foi realizado na estação de Piscicultura da Universidade Estadual de Londrina - Paraná. O município localiza-se a $23^{\circ} 23^{\prime}$ de latitude sul e $51^{\circ} 11$ 'de latitude oeste, com altitude média de 566 metros. O clima, segundo a classificação de Köppen, é do tipo cfa, ou seja sub-tropical úmido. A classificação do solo no local do experimento, de acordo com Rocha, Barros e Guimarães (1991), é terra roxa estruturada latossolisada eutrófica A moderada com textura argilosa.

A amostra foi constituída de 65 carpas (Cyprinus carpio), sendo 34 fêmeas e 31 machos oriundos da mesma desova, criados em um viveiro de $100 \mathrm{~m}^{2}$, recebendo ração para crescimento com $30 \%$ de proteína bruta e $2.800 \mathrm{Kcal}$ de energia metabolizável e identificados com marcação a ferro incandescente, em idade entre 60 e 240 dias. As características avaliadas a partir da idade de 60 dias, e a cada 60 dias de intervalo até a idade de 240 dias, foram: peso corporal $(\mathrm{g})$ e o comprimento corporal total $(\mathrm{cm})$.

A qualidade da água foi determinada através de amostragem a cada 15 dias para as características: temperatura, $\mathrm{pH}$, condutividade elétrica e oxigênio dissolvido.

O fator de condição alométrico e o de Fulton foi calculado da seguinte forma: inicialmente o peso e o comprimento total foram transformados para logaritmo natural. Em seguida, foram determinados o A (intercepto) e o B (coeficiente de regressão). Como esta relação é do tipo exponencial $\left(y=a \cdot x^{b}\right)$, 
o coeficiente de regressão calculado para dados transformados é igual ao coeficiente de regressão para essa relação.

No entanto, o intercepto calculado através da transformação não apresenta a constante da relação exponencial, o cálculo é feito através de:

$$
\mathrm{a}=\mathrm{e}^{-\mathrm{A}}
$$

Em que:

$\mathrm{a}=$ intercepto $1_{\mathrm{n} \text { a }}$

$\mathrm{e}=$ antilogarítmo natural

A = intercepto calculado com a transformação das variáveis para $1_{n}$

O fator de condição de Fulton, foi calculado utilizando-se da relação:

$$
\mathrm{K}_{\mathrm{f}}=\mathrm{W} / 1^{3}
$$

Em que:

$\mathrm{K}_{\mathrm{f}}=$ fator de condição de Fulton

$\mathrm{W}=$ peso corporal $(\mathrm{g})$

$1=$ comprimento corporal total $(\mathrm{cm})$

O fator de condição alométrico foi calculado substituindo a constante $(3,0)$ pelo valor do coeficiente de regressão, sendo o seguinte:

$$
\mathrm{K}_{\mathrm{A}}=\mathrm{W} / \mathrm{l}^{\mathrm{b}}
$$

Em que:

$\mathrm{K}_{\mathrm{A}}=$ fator de condição alométrico

$\mathrm{W}=$ Peso corporal $(\mathrm{g})$

$1=$ comprimento corporal total $(\mathrm{cm})$

$\mathrm{b}=$ coeficiente de regressão

A taxa de crescimento médio (igr \%) entre as médias dos pesos corporais em idade de 60, 120, 180 e 240 dias separados por sexo, foi determinada através da fórmula apresentada por Ricker (1979).

$$
\operatorname{Igr}=\left(1_{n} w_{f}-1_{n} w_{i}\right) /\left(t_{f}-t_{i}\right)
$$

Em que:

$1_{\mathrm{n}} \mathrm{w}_{\mathrm{f}}=$ logarítmo natural do peso na idade considerada $1_{\mathrm{n}} \mathrm{W}_{\mathrm{i}}=$ logarítmo natural do peso na idade anterior $\mathrm{t}_{\mathrm{f}}=$ idade final

$\mathrm{t}_{\mathrm{i}}=$ idade inicial

\section{Resultados e Discussão}

Durante o período experimental, a qualidade da água apresentou os seguintes resultados, conforme os parâmetros avaliados e que constam na Tabela 1 .

Tabela 1. Número de avaliação $(\mathrm{N})$, média, desvio padrão (s) e coeficiente de variação $(\mathrm{CV})$ para as características: Temperatura, $\mathrm{pH}$, condutividade elétrica e oxigênio dissolvido, avaliados no viveiro de criação.

\begin{tabular}{ccccc}
\hline Característica & $\mathrm{N}$ & Média & $\mathrm{s}$ & $\mathrm{CV}(\%)$ \\
\hline Temperatura $\left({ }^{\circ} \mathrm{C}\right)$ & 12 & 22,42 & 2,56 & 10,79 \\
$\mathrm{pH}$ & 12 & 6,82 & 0,50 & 7,26 \\
$\begin{array}{c}\text { Condutividade elétrica } \\
\left(\mu \mathrm{scm}^{-1}\right)\end{array}$ & 12 & 59,08 & 6,73 & 11,38 \\
$\begin{array}{c}\text { Oxigênio dissolvido }\left(\mathrm{mg} . \mathrm{l}^{-1}\right) \\
\text { (i) }\end{array}$ & 12 & 6,75 & 0,62 & 9,20 \\
\hline
\end{tabular}


Os valores da Tabela 1 indicam que estes estão dentro do limite apresentado por Pádua (1994). Desta forma, as condições ambientais representadas pelos parâmetros avaliados na água do viveiro de criação foram compatíveis durante todo o experimento, com valores recomendados para a manutenção de organismos aquáticos de água doce.

No Gráfico 1, são apresentados o crescimento de machos e fêmeas no período de 60 a 240 dias de idade.

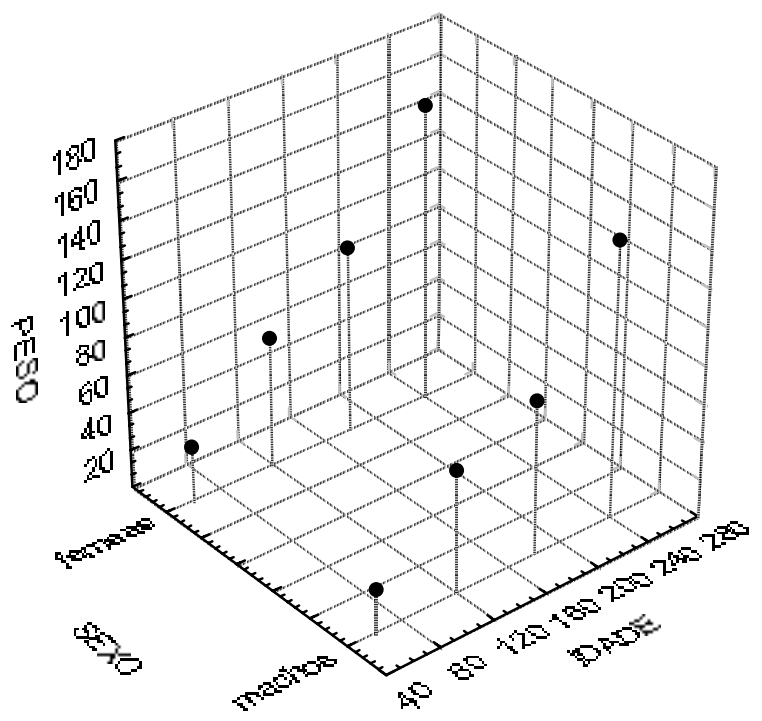

Gráfico 1. Peso corporal de carpa (Cyprinus carpio) considerando os sexos e idades

Este gráfico indica que nesta espécie o crescimento foi maior nas fềmeas do que nos machos. O dimorfismo sexual foi evidenciado por El-Ibiary e Joyce (1978) no catfish (Ictalurus punctatus) para a característica peso aos 336 dias de idade. Esta diferença é segundo
Dunham e Smitherman (1983), devido ao maior desenvolvimento dos machos nesta espécie.

Campton e Gall (1988), trabalhando com Gambusia affinis não encontraram diferenças significativas, $(p>0,05)$ no comprimento corporal nas idades de 21 a 28 dias, mas aos 42 e 52 dias, os machos tinham um tamanho significativamente menor $(\mathrm{p}<0,01)$.

No salmão do atlântico (Salmo salar), Gjerde e Gjedrem (1984) encontraram diferenças significativas $(p<0,05)$ entre machos e fềmeas, sendo os machos mais desenvolvidos do que as fêmeas. Resultados semelhantes foram obtidos por Siitonen (1986) em truta arco-íris (Salmo gairdneri) na idade de dois anos e meio, sendo o peso dos machos superiores aos das fêmeas. No entanto, esta superioridade não foi significativa $(p>0,05)$.

Embora nesta espécie (Cyprinus cárpio) os machos crescem menos do que as fêmeas, não foi encontrado diferenças estatísticas significativas entre os sexos $(p>0,05)$, nesta faixa etária. Esta diferença é mais evidente em peixes adultos em que as fêmeas, apresenta uma maior reserva de energia para o momento da desova, conforme (SHULMAM; LOVE, 1999).

Na Tabela 2 são apresentados os valores do intercepto, coeficiente de regressão, coeficiente de determinação e o tipo de crescimento para a relação peso corporal e comprimento corporal, conforme os sexos, na carpa.

Tabela 2. Valores do intercepto (lna), coeficiente de regressão (b), coeficiente de determinação ( $\mathrm{r}^{2}$ ) e o tipo de crescimento corporal, conforme os sexos na carpa.

\begin{tabular}{ccccc}
\hline Sexos & $\operatorname{lna}$ & $\mathrm{b}$ & $\mathrm{r}^{2}$ & cresc. \\
\hline Machos & 0,023988 & 2,90 & 0,97 & Alométrico \\
Fêmeas & 0,029974 & 2,82 & 0,94 & Alométrico \\
\hline
\end{tabular}


A relação entre o peso e comprimento total apresentou crescimento alométrico negativo $(\mathrm{P}<0,05)$ para os machos $(b=2,90)$ e fêmeas $(b=2,82)$. O coeficiente de determinação $\left(\mathrm{r}^{2}\right)$ indica que a variação do peso corporal é explicada pela quase totalidade $(90 \%)$ da variação do comprimento corporal, para ambos os sexos. Este valor está dentro da faixa de valores citados por Le Cren (1951) e Royce (1972) e próximo aos valores obtidos por Scharf et al. (1998) que determinaram a relação peso corporal e comprimento corporal total em 10 espécies de peixes marinhos, sendo que o valor de b oscilou entre 2,99 a
3,45 , com o coeficiente de determinação ao redor de $90 \%$. Os valores encontrados também estão próximos daqueles relatados por Gunnes e Gjedrem (1981); Gjerde e Schaffer (1989) na truta arco-iris (Salmo gairdneri); Refstie e Steine (1978) no salmão e Rocha (1995) no curimbatá (Prochilodus lineatus). O resultado indica que o peso teve um incremento maior do que o comprimento corporal total, o que concorda com o determinado por Rocha (1995).

Na Tabela 3, são apresentados o fator de condição alométrico e o de Fulton separado por sexo e idade.

Tabela 3. Valores do fator de condição alométrico $\left(\mathrm{F}_{\mathrm{A}}\right)$ e de Fulton $\left(\mathrm{F}_{\mathrm{F}}\right)$ em carpas (Cyprinus carpio), considerando o sexo e a idade.

\begin{tabular}{cccccc}
\hline Idade (dias) & \multicolumn{3}{c}{ Machos } & & \multicolumn{2}{c}{ Fêmeas } \\
& $\mathrm{F}_{\mathrm{A}}\left(10^{2}\right)$ & $\mathrm{F}_{\mathrm{F}}\left(10^{2}\right)$ & & $\mathrm{F}_{\mathrm{A}}\left(10^{2}\right)$ & $\mathrm{F}_{\mathrm{F}}\left(10^{2}\right)$ \\
\cline { 2 - 3 } \cline { 5 - 6 } 60 & 2,622 & 2,072 & & & 2,741 \\
120 & 2,529 & 1,929 & & 2,475 & 1,937 \\
180 & 2,441 & 1,845 & & 2,517 & 1,949 \\
240 & 2,568 & 1,907 & & 2,419 & 1,845 \\
\hline
\end{tabular}

Os resultados indicam que o fator de condição alométrico e de Fulton apresentaram diferenças no desenvolvimento de machos e fêmeas. O desenvolvimento nas fêmeas foi maior que nos machos, a exceção na idade de 120 dias.

Nos machos foi observado ainda uma queda maior no fator de condição em idade entre 120 e 180 dias, o que não ocorreu com as fêmeas, que foi mais uniforme. No entanto, os machos se recuperaram na idade de 240 dias.

Os resultados mostram que o fator de condição alométrico e o de Fulton mostraram diferenças entre os sexos e dentro de sexos. No entanto, o fator de condição alométrico evidenciou melhor as diferenças entre as idades, nos dois sexos. $\mathrm{O}$ fator de condição de Fulton embora tenha apresentado diferenças entre as idades nos dois sexos, estas foram bem menores do que as observadas através do fator de condição alométrico.

O uso do fator de condição alométrico exige que se tenha o peso corporal e comprimento corporal total nas várias idades para o cálculo do coeficiente de regressão (b), o que nem sempre está disponível. Desta forma, em estudos de peixes na natureza o fator de condição de Fulton é o mais apropriado, pois utiliza o coeficiente de regressão é 3,0 (isométrico) conforme Ratz e Lloret (2003), Lloret et al. (2002) e Halliday (2001).

Na Tabela 4 são apresentados a taxa de crescimento médio (igr (\%)).

Tabela 4. Taxa de crescimento médio (igr (\%)) conforme a idade e sexo na carpa (Cyprinus carpio).

\begin{tabular}{|c|c|c|}
\hline \multirow[t]{2}{*}{ Idade (dias) } & \multicolumn{2}{|c|}{$\operatorname{Igr}(\%)$} \\
\hline & Machos & Fêmeas \\
\hline 60 & - & - \\
\hline 120 & 1,61 & 1,57 \\
\hline 180 & 0,31 & 0,42 \\
\hline 240 & 0,87 & 0,91 \\
\hline
\end{tabular}


Os resultados da Tabela 3 indicam o maior desenvolvimento das fêmeas em várias idades, a exceção em idade entre 60 e 120 dias. A menor taxa ocorrida em idade entre 120 e 180 dias em ambos os sexos coincidiu com o período de inverno com temperatura média de $17^{\circ} \mathrm{C}$.

\section{Conclusões}

As fêmeas apresentaram crescimento maior do que os machos quando comparado pela taxa de crescimento médio. A comparação dos peixes pelo fator de condição Fulton e alométrico, apresentou um bom parâmetro para identificar o desenvolvimento dos animais de mesma espécie, criados em condições iguais ou diferentes.

Na espécie Cyprinus carpio, a comparação entre os animais pode ser feita com o fator de condição, sendo alométrico o mais indicado.

\section{Referências}

CAMPTON, D. E.; GALL, G. A. E. Responses to selection for body size and age at sexual maturity in the mosquito fish (Gambusia affimis). Aquaculture, Amsterdam, v. 68, n. 3, p. 221-241, 1988.

DUNHAM, R. A.; SMITHERMAN, R. O. Response to selection and realized heritability for body weight in 3 strains of channel catfish (Ictalurus punctatus), Grow in Earthern Ponds. Aquaculture, Amsterdam, v. 33, p. 89-96, 1983.

EL IBIARY,H. M.; JOYCE, J. A. Hertability of body size traits, dressind weight and lipid content in channel catfish. Journal of Animal Science, Champaign, v. 47, p. 82-88, 1978.

GJERDE, B.; GJEDREM, T. Estimates of phenotypic and genetic parameters for carcass traits in atlantic salmon and raibow trout. Aquaculture, Amsterdam, v. 36, p. 97$110,1984$.

GJERDE, B.; SCHAEFFER,L.R. Body traits in rainbow trout II- Estimates of heritabilities and of phenotypic and genetic correlations. Aquaculture, Amsterdam, v. 80, p. 25-44, 1989.

GUNNES,K.; GJEDREM,T. A genetic analysis of body weigth and length in rainbow trout reared in seawater for 18 months. Aquaculture, Amsterdam, v. 24, p. 161-174, 1981.
HALLIDAY, R. G. Proceedings of the fisheries management studies working group. Bedford Institute of Oceanography, Dartmouth, 2001. 82 p.

LE CREN, E. D. The lenght-weight relationship and seasonal cycle in gonad weight and condition in the perch (Perca fluviatis). Journal of Animal Ecology, Oxford, v. 20, p. 201-219, 1951.

LLORET, J.; DE SOLA, L. G.; SOUPLET, A.; GALZIN, R. Effects of large-scale habitat variability on condition of demersal exploited fish in the North-Western Mediterranean. Journal of Marine Science, Dauphin, v. 59, p. 1215-1227, 2002.

PADUA, H. B. Teores Recomendados para Manutenção de Organismos Aquáticos de Água Doce. Panorama da Aqüicultura, Rio de Janeiro, v. 4, n. 21, p. 8-9, 1994.

RATZ, H. J.; LLORET, J. Variation in fish condition between atlantic cod (Gadus morhua) atocks the effect on their productivity and management implications, Fisheries Rescarch, Amsterdam, v. 60, p. 369-380, 2003.

REFSTIE, T.; STEINE, T. Selection experiments with salmon. III- Genetic and environmental sources of variation in length and weigth of atlantic salmon in the fresh water phase. Aquaculture, Amsterdam, v. 14, p. 221-234, 1978.

RICKER, W. E. Growth rates and models. In: HOAR, W. S; RANDALL, D. J.; BRETT, J. R. (eds) Fish physiology bioenergetics and growth. New York: Academic Press, 1979. $786 \mathrm{p}$.

ROCHA, M. A. Estimativas de herdabilidade e correlações genéticas, fenotípicas e ambientais de características avaliadas no curimbatá (Prochilodus liveatus) em idades entre 60 e 330 dias. 1995. 150 f. Tese (Doutorado em Aquicultura) - Centro de Aquicultura, Universidade Estadual Paulista, Jaboticabal, 1995.

ROCHA, G. C.; BARROS, O. N. F.; GUIMARÃES, M. F. Distribuição espacial e características dos solos do campus da Universidade Estadual de Londrina-Pr. Semina, Londrina, v. 12, p. 25-27, 1991.

ROYCE, W. F. Introduction to the fishery science. New York: Academic Press, 1972.351 p.

SCHARF, F. S.; YETTER, R. M.; SUMMERS, A. P.; JUANES, F. Enhancing diet analyses of piscivorous fishes in the northwest atlantic through identification and reconstruction of original prey sizes from ingested remains. Fishery Bulletin, Seattle, v. 96, n. 3, p. 575-588, 1998.

SHULMAN, G. E.; LOVE, R. M. The bioquemical ecology of marine fishes. In: SOUTHWARD, A. J.; TAYLER, P. A.; YOUG, C. M. (eds.) Advances in Marine ecology. London: Academic Press, 1999. 351 p.

SIITONEN, L. Factors affecting growth in rainbow trout (Salmo gairdneri). Stocks. Aquaculture, Amsterdam, v. 57, p. 185-191, 1986. 